\title{
In-situ TEM Observation of Pt-terminating Carbyne on Graphene
}

\author{
Emi Kano ${ }^{1,4}$, Ayako Hashimoto ${ }^{2,3,4}$ and Masaki Takeguchi ${ }^{1,2,4}$ \\ 1. Institute of Applied Physics, University of Tsukuba, Tsukuba, Japan. \\ 2. Surface Physics and Structure Unit, National Institute for Materials Science, Tsukuba, Japan. \\ 3. Global Research Center for Environment and Energy based on Nanomaterials Science, National \\ Institute for Materials Science, Tsukuba, Japan. \\ 4. Transmission Electron Microscopy Station, National Institute for Materials Science, Tsukuba, Japan.
}

Carbyne is a one-dimensional (1D) single atomic linear chain, composed of sp-hybridized carbon atoms. Some low-dimensional carbon allotropes, such as zero-dimensional (0D) fullerenes, quasi-onedimensional (quasi-1D) carbon nanotubes (CNTs), and two-dimensional (2D) graphene have recently been discovered and have attracted worldwide attention for their unique properties. Theoretical studies have predicted that carbynes have more remarkable properties than these materials: twice the tensile stiffness of graphene and CNT, a tension-induced increase in its electronic band gap, high stability at room temperature, and so on [1]. However, these properties have not yet been proven experimentally because of the difficulties encountered in production and during observation.

Many methods for the production of carbynes have been demonstrated so far. For example, finite-length carbon chains have been chemically synthesized in solution, but it is difficult to determine their exact structures and properties only by spectroscopic analysis. Some researchers synthesized carbynes and observed their atomic structure by in situ transmission electron microscopy (TEM) [2]. They utilized an electron-beam irradiation technique to etch the graphene sheets inside TEM equipment. This method requires very careful control of the beam-irradiation conditions to produce only a small number of carbynes. Hence, the method can only be used for fundamental studies concerning the atomic structure of carbyne and carbon-chain bonding, but it is not suitable for application purposes.

Here, we report a novel, reproducible method of carbyne formation using Pt atoms on graphene. The formation and dynamics of carbynes were observed on an atomic scale by aberration-corrected TEM (JEM-ARM200F, JEOL). The samples were obtained by transferring monolayer graphene membranes onto in situ heating chips (E-chips for Aduro, Protochips). Pt was deposited by a plasma sputtering system. We observed independent Pt atoms that appeared on a clean graphene surface. The accelerating voltage was $80 \mathrm{kV}$, and we observed the migrations of $\mathrm{Pt}$ and $\mathrm{C}$ atoms on the graphene surface at $400{ }^{\circ} \mathrm{C}$ with an in situ heating holder (Aduro heating holder, Protochips).

Figure 1A-C show TEM images of carbyne formation. Three Pt atoms captured some carbon atoms, resulting in the formation of a $\mathrm{C}$-shape chain (Fig. 1A). The Pt atoms and the chain moved around freely for $1 \mathrm{~min} 22 \mathrm{~s}$, and then the chain suddenly turned into a straight chain (Fig. 1C). Both ends of the chain were terminated by $\mathrm{Pt}$ atoms, and the chain remained motionless for more than $20 \mathrm{~s}$. Carbynes are linear chains, while hydrocarbon chains such as alkane $\left(\mathrm{C}_{n} \mathrm{H}_{2 n+2}\right)$ and polyacetylene $(\mathrm{CH})_{n}$ have zigzag chain structure with bonding angle of $120^{\circ}$ and $109.5^{\circ}$, respectively. In our experiment, no zigzag chain structure was observed. Therefore, we deduced that the observed structures were carbynes. From Fig. 1C, the length between the $\mathrm{Pt}$ atoms at both ends was measured to be approximately $1.5 \mathrm{~nm}$. It corresponds to a model composed of 11 carbons with two Pt atoms (Fig. 1D). Fig. 1E shows a simulated image using this model. 
Using independent $\mathrm{Pt}$ atoms is the most important key to produce and stabilize carbyne chains. The Pt atoms we report here are not clustered atoms. Figure 2 illustrates a following bottom-up process of carbyne formation we observed. First, free carbon adatoms on graphene were trapped by the Pt atoms, which served as nucleation sites for carbyne formation (Fig. 2A). Then, the carbon atoms connected to form a C-shape chain (Fig.2B). The C-shape chain and the Pt atoms still fluctuated. It is presumed that the bond between $\mathrm{Pt}$ and $\mathrm{C}$ atom was weak at that situation. Finally, all the carbyne chains became stable and came to rest in a straight shape (Fig. 2C). Once a carbyne chain was terminated by Pt atoms at both ends, it couldn't grow by combining with carbon atoms anymore. If the Pt atoms act as a catalyst, the length of the chain would change like a CNT synthesis (Pt nano particles act as a catalyst for the growth of CNTs). However, the Pt atoms did not play such a role. Thus, we conclude the Pt atoms in our study act as nucleation sites and serve to terminate the ends of carbynes, but not act as a catalyst.

\section{References:}

[1] M. Liu et al, ACS Nano 7 (2013), 10075-10082.

[2] A. Chuvilin et al, New J. Phys. 11 (2009),

083019.

[3] The authors acknowledge discussions with Dr. J. Fujita and Dr. J. Nakamura. A part of this work was supported by "Nanotechnology Platform Project" of the Ministry of Education, Culture, Sports, Science and Technology (MEXT), Japan.
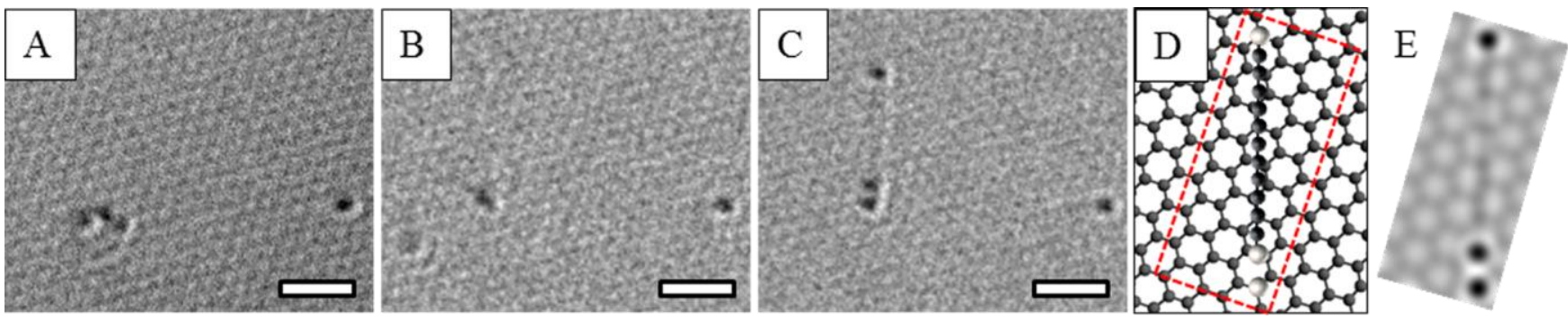

Figure 1. (A to C) TEM images of carbyne chain, which exhibited a variety of ringed, curved, straight shapes. Scale bars are $1 \mathrm{~nm}$. (D) Atomic model of straight carbyne. (E) Simulated TEM image.
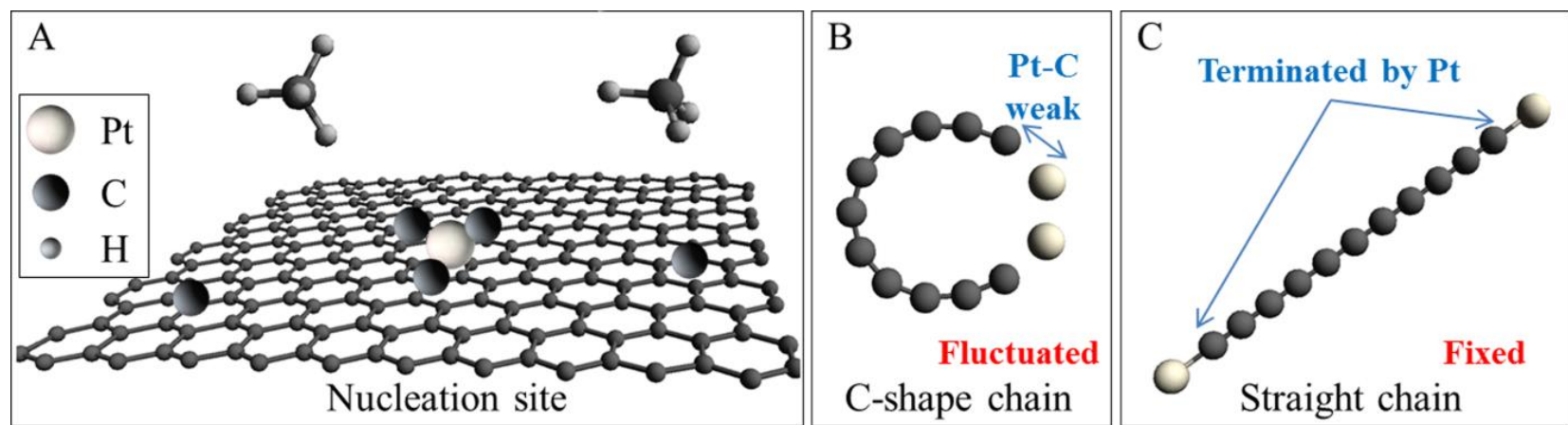

Figure 2. Schematic illustrations of a bottom-up process of formation of carbyne with $\mathrm{Pt}$ atoms. 